\title{
Neglected simultaneous bilateral femoral neck fractures secondary to narcotic drug abuse treated by bilateral one-staged hemiarthroplasty: a case report
}

\author{
Alireza Hootkani, Ali Moradi and Ehsan Vahedi*
}

\begin{abstract}
Simultaneous bilateral femoral neck fractures are extremely rare and associated with various conditions. Up to now Most cases had correlations with major trauma, repetitive minor trauma, seizure, parathyroid or renal dysfunction, antiepileptic medications, seizure, etc. A 28 -year-old addict man referred to us with a 10-year history of narcotic drug abuse and history of 8 months bilateral groin pain. He admitted with displaced bilateral femoral neck fracture. Because of long duration of this condition and osteonecrosis revealed on bone scan, one-staged bilateral hip hemiarthroplasty was done. A good function was noted after surgery to 4-month follow up. Up to now, have not be founded in the literature that a case of bilateral femoral neck fracture associated with narcotic drug abuse.

Because of negative effects of opium or smoking on bone tissues, a simple bone pain should aware us about the risk of stress or fatigue fracture.
\end{abstract}

\section{Background}

Simultaneous bilateral femoral neck fractures are extremely rare and have been associated with highenergy trauma [1], repetitive minor trauma, abnormal anatomy [2], seizure [3-9], electrical injury, electroconvulsive therapy, primary or secondary bone diseases such as osteomalacia [10], hyperparathyroidism [11], chronic renal failure [12], or severe osteoporosis especially after corticosteroid or other corticosteroid-like drug therapy.

Different procedures have reported in the literature for treatment of bilateral femoral neck fracture include in situ fixation, open reduction and internal fixation, open fixation with valgus intertrochanteric osteotomy [13] and hemi or total hip arthroplasty in one [14] or two-staged operations.

The complications include: non-union, delayed union and shortening. Femoral head osteonecrosis and coxa vara can be avoided with correct treatment.

In this report, we present a case of bilateral femoral neck fractures with prolonged history of narcotic drug

* Correspondence: e_vahedi@yahoo.com

1 Orthopaedics division, Emamreza Medical Center, Mashhad University of Medical Sciences, Mashhad, Iran

Full list of author information is available at the end of the article abuse, treated by bilateral hemiarthroplasty. We did not found any previously recognized underlying risk factors for stress fracture.

\section{Case presentation}

A 28-yr-old addict man was referred to our institution with an 8 month history of chronic pain in both groins aggravated by weight bearing sometimes keeping him awake at night. He had previously normal activities without specific sport or job program. By disease onset, His activities were restricted because of the pain and decreased range of motion. About 4 months before operation, the patient was visited by a physician who took a plain pelvic radiograph which revealed displaced left and non-displaced right femoral neck stress fracture (Fig-1).

After 8-month, because of low socio-economic state and not appropriate follow-up by him, finally, the patient was referred to us with displaced bilateral hip fractures (Fig-2).

On examination, his weight was 65 kilograms and both active and passive motions were painful and restricted. Active straight leg raise was not possible on either side. 


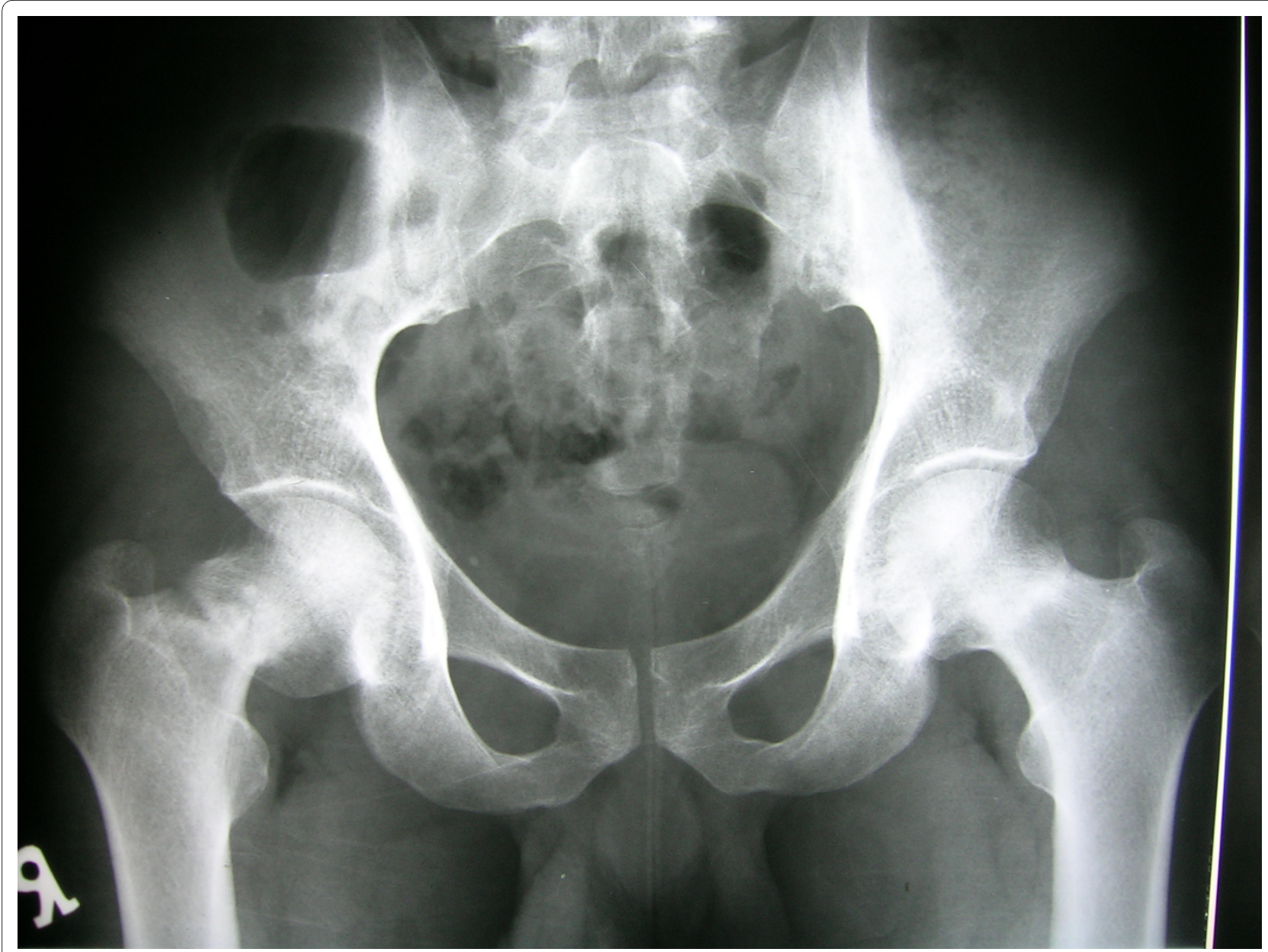

Figure 1 Radiograph taken 4 months before operation demonstrates displaced left femoral neck stress fracture and non-displaced right femoral neck stress fracture.

No limb-length discrepancy was observed because of symmetrical nature of the deformity.

There was no evidence of any underlying metabolic or endocrine disease affecting the bone strength in physical examination or family history.

In laboratory tests, CBC, ESR, alkaline phosphatase, serum calcium, phosphorus and serum total protein levels were normal. Normal Parathyroid, thyroid, renal, gonadal and liver function tests were detected. 24-hour urinary excretion for calcium and phosphorus was also within normal ranges.

Because of long period of immobilization and lesser activities, which decreased bone density, we did not consider bone densitometry for him.

Skeletal bone scan with TC-99m showed no other abnormal findings except bilateral femoral head osteonecrosis and bilateral femoral neck fracture (Fig-3).

Surgery was performed using general anesthesia with the patient lateral on a conventional operating table for both right and left hips at one section choosing postero- lateral minimal incision approach. The cartilage of the acetabulum was intact and heads of the femurs were decided to be replaced with bipolar prosthesis (Fig-4). During operation, it was noted that bone ends at the fracture site was extremely sclerotic and difficult to penetrate with instruments. A biopsy showed no evidence of metabolic bone disease and osteonecrosis was reported. The postoperative period was uneventful. Quadriceps exercises and pain free movement of both hips were permitted from the first post operative day. Then, the patient was permitted to bear weight.

The patient had normal range of motion in both hips and was capable of doing daily activities after 24 weeks of follow up.

\section{Discussion}

Hip Stress fractures of most frequently involve the medial aspect of femoral neck. They are termed fatigue fracture in patients who have normal bone but are exposed to excessive mechanical stress, such as many seen in athletes 


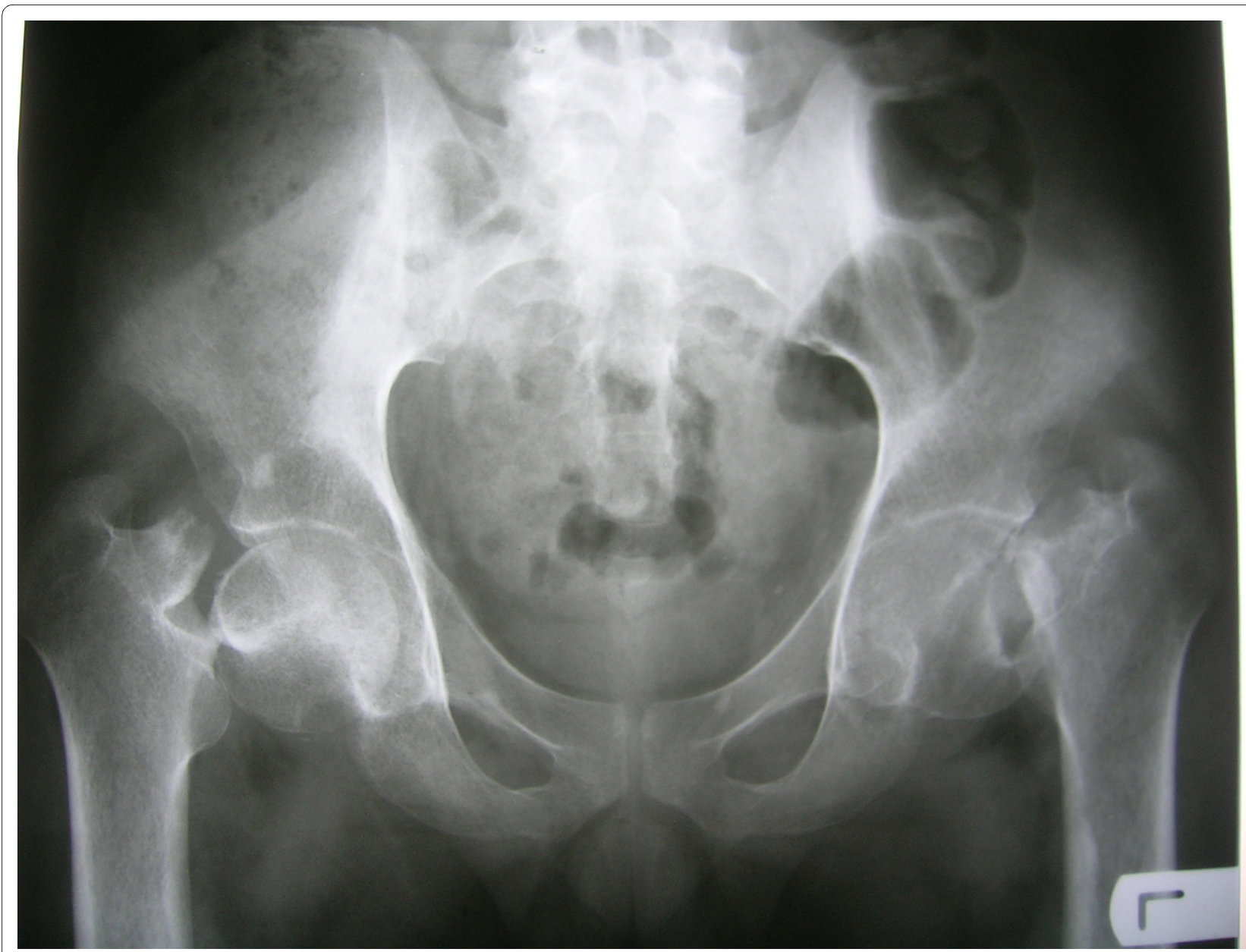

Figure 2 Radiograph taken before operation demonstrates displaced bilateral femoral neck stress fractures.

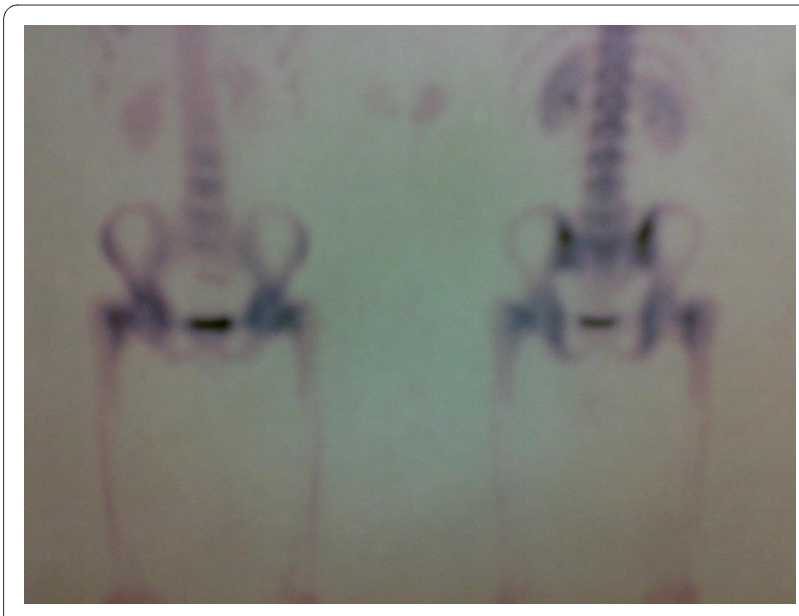

Figure 3 Skeletal bone scan with TC-99m showed no other abnormal findings except bilateral femoral head osteonecrosis and bilateral femoral neck fracture.

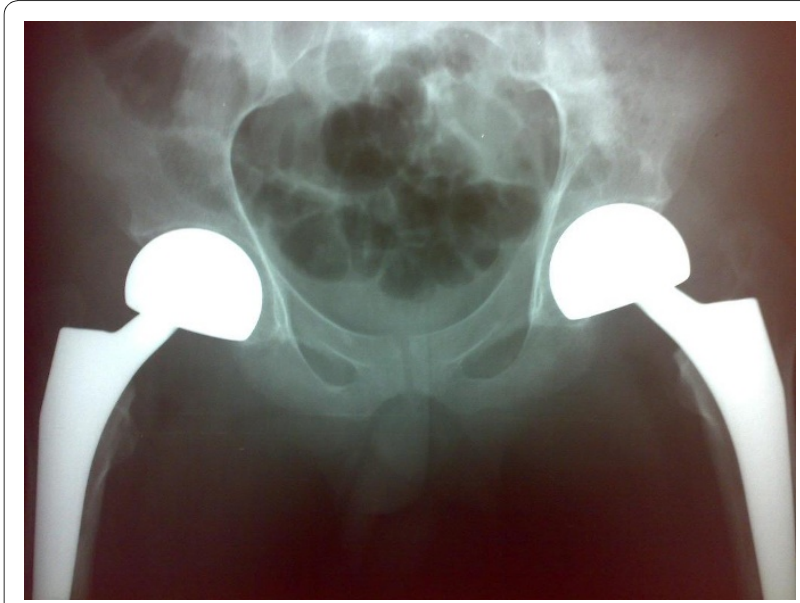

Figure 4 Radiograph taken after operation demonstrates replacement of left and right hip with bipolar prosthesis. 
and army recruits, and they are termed insufficiency fractures in patients who have abnormal bone subjected to normal stress.

The remarkable characteristics of this case include simultaneous, bilateral, displaced fractures with no evidence of underlying pathologic factor; suspicious relationship with narcotic drug abuse and simultaneous operation of both hips in one section with minimal incision approach.

In this case, stress fractures of femoral neck were probably related to 10 year history of drug abuse. By further abuse, the pain decreased and continuity of activities aggravated the fracture and ultimately complete bilateral femoral neck fracture occurred.

Many procedures have been reported in the literature for treatment of bilateral femoral neck fractures. These include lag screw or dynamic hip screw fixation [6$8,15,16]$, pedicle bone grafting [10], and a combination of these. In this case, because of the complete displacement and osteonecrosis and high risk of nonunion (23\%-43\%) [17-19], we decided to replace the femoral head instead of open reduction and internal fixation, and young age of the patient did not change our decision.

To our knowledge, this is the first report in the English literature of bilateral neglected displaced femoral neck fractures in which the only predisposing factor was narcotic drug abuse.

\section{Conclusions}

The negative effects of smoking or opium on bone tissues or bone healing processes were well recognized. Also, narcotic agents diminish signs and symptoms of any painful condition affecting the bones. Because of various risk factors predisposing many people to development of stress fractures, we suggest that each patient who complaints of prolonged bone pain and has positive history of opium abuse, should be considered an important underlying bone disease such as stress fracture until proven otherwise.

\section{Consent}

Written informed consent was obtained from the patient for publication of this case report and any accompanying images. A copy of written consent is available for review by the editor-in-chief of this journal.

\section{Competing interests}

The authors declare that they have no competing interests.

\section{Authors' contributions}

AH performed part of the literature review, examined the patient and operated him. Also he had given final approval of the version to be published and generally supervised this report. AM also performed part of literature review, participated in operation and conceived the idea of present study and case report presentation. EV contributes in literature review, analyzed the data and involved in editing the manuscript. All authors have read and approved the final manuscript.

\section{Author Details}

Orthopaedics division, Emamreza Medical Center, Mashhad University of Medical Sciences, Mashhad, Iran

Received: 1 May 2010 Accepted: 25 June 2010

Published: 25 June 2010

\section{References}

1. Schroder J, Marti RK: Simultaneous bilateral femoral neck fractures: case report. Swiss Surg 2001, 7:222-224

2. Annan $\mid \mathrm{H}$, Buxton RA: Bilateral stress fractures of the femoral neck associated with abnormal anatomy: a case report. Injury 1986, 17:164-166

3. Funk L, Faux JC: Bilateral combined femoral neck and acetabular fractures due to cerebro-vascular ischemic induced convulsion. Eur J Orthop Surg Traumatol 1998, 8:145-146.

4. Gur S, Yilmaz H, Tuzuner S: Fractures due to hypocalcemic convulsion. Int Orthop 1999, 23:308-309.

5. Kause J, Parr MJ: Bilateral subcapital neck of femur fractures after eclamptic seizures. B J Anesth 2004, 92:590-592.

6. Powell HDW: Simultaneous bilateral fractures of the neck of the femur. J Bone Joint Surg 1960, 42(B):236-252.

7. Rahman MM, Awada A: Bilateral simultaneous hip fractures secondary to an epileptic seizure. Saudi Med J 2003, 24:1261-1263.

8. Suh KT, Kang DJ, Lee JS: Bilateral intertrochanteric fractures after surgical treatment of bilateral femoral neck fractures secondary to hypocalcemic convulsions with chronic renal failure: a case report and review of the literature. Arch Orthop Trauma Surg 2006, 126:123-126.

9. Tompkins GS, Henderson RC, Peterson HD: Bilateral simultaneous fractures of the femoral neck: case report. J Trauma 2002, 30:415-1416.

10. Chadha M, Balain B, Maini L: Spontaneous bilateral displaced femoral neck fractures in nutritional osteomalacia-a case report. Acta Orthop Scand 2001, 72:94-96.

11. Chen $\mathrm{CE}$, Kao CL, Wang CJ: Bilateral pathological femoral neck fractures secondary to ectopic parathyroid adenoma. Arch Orthop Trauma Surg 1998, 118:164-166.

12. Madhok R, Rand JA: Ten year follow-up study of missed, simultaneous, bilateral femoral-neck fracture treated by bipolar arthroplasties in a patient with chronic renal failure. Clin Orthop 1993, 291:185-187.

13. Narender M K, Rajesh R, Gulia AD D, Roop S: Valgus intertrochanteric osteotomy in neglected simultaneous, bilateral, displaced subcapital femoral neck fractures in an epileptic pregnant woman. Current Orthopedic Practice 2009, 20:461-463.

14. Grimaldi M, Vouaillat $H$, Tonetti J, Merloz P: simultaneous bilateral femoral neck fractures secondary to epileptic seizures: treatment by bilateral total hip arthroplasty. Rev Chir Orthop Traumatol 2009, 95(7):555-7.

15. Haddad FS, Mohanna PN, Goddard NJ: Bilateral femoral neck stress fractures following steroid treatment. Injury 1997, 28:671-673.

16. Kalci A, Yanat AN, Sevinc T: Insufficiency fractures of both femoral necks in a young adult caused by osteoporosis: a case report. Arch Orthop Trauma Surg 2008, 128(8):865-8.

17. Jackson M, Learmonth ID: The treatment of nonunion after intracapsular fracture of proximal femur. Clin Orthop 2002, 399:1 19-128.

18. LaVelle DG: Fractures and dislocations of the hip. Edited by: Canale ST. Campbell's Operative Orthopedics. Philadelphia: Mosby-Elsevier; 2008:3237-3308.

19. Lu-Yao GL, Keller RB, Littenberg B: Outcomes after displaced fractures of the femoral neck: a meta-analysis of one hundred and six published reports. J Bone Joint Surg 1994, 76(A):15-25.

doi: 10.1186/1749-799X-5-41

Cite this article as: Hootkani et al., Neglected simultaneous bilateral femoral neck fractures secondary to narcotic drug abuse treated by bilateral onestaged hemiarthroplasty: a case report Journal of Orthopaedic Surgery and Research 2010, 5:41 